\title{
Probing Structure/Property Relationships of Ce-rich Oxygen Evolution Catalysts by Advanced Transmission Electron Microscopy
}

\author{
C. Kisielowski ${ }^{1,2}$ J.A. Haber ${ }^{3}$, J.M. Gregoire ${ }^{3}$, Y. Cai ${ }^{2}$
}

1. Joint Center for Artificial Photosynthesis (JCAP), Lawrence Berkeley National Laboratory, 1 Cyclotron Road, Berkeley, CA 94720 (USA).

2. National Center for Electron Microscopy, Lawrence Berkeley National Laboratory, 1 Cyclotron Road, Berkeley, CA 94720 (USA).

3. Joint Center for Artificial Photosynthesis (JCAP), MC 132-80, 1200 E. California Blvd. Pasadena, CA 91125 (USA)

A new family of Ce-rich catalysts composed of earth abundant elements was recently discovered in JCAP using their high-throughput methods [1]. These most active OER catalysts are composed of quaternary alloys such as $\mathrm{Ni}_{0.3} \mathrm{Fe}_{0.07} \mathrm{Co}_{0.20} \mathrm{Ce}_{0.43} \mathrm{O}_{\mathrm{x}}$ and fabricated by printing and successive annealing steps. Alternatively they can be grown by electrodeposition. However, their local chemical composition and atomic structure are unknown since they are composed of small particles that often appear "x-ray amorphous". Moreover, previously unavoidable beam-sample interactions can rapidly alter their structure and chemical composition. The application of electron in-line holography with variable voltages and dose rates [2] removes these limitations and allows studying the static or dynamic behavior of crystalline and amorphous catalysts in real time with single atom sensitivity [3].

The Figure 1a) and shows flakes of the printed catalyst at low and at high magnification. The catalyst was prepared by depositing metal nitrate precursors on a pyrex petri dish followed by calcination in air at $350 \mathrm{C}$ for $10 \mathrm{~h}$. In Figure 1d) the material is prepared by printing on a glassy carbon support. The atomic resolution images reveal a random agglomeration of sintered grains with a diameter that is smaller than $3 \mathrm{~nm}$. An indexing of its Fourier transform (Figure 1b) is compatible with the presence of $\mathrm{CeO}_{2}$ and $\mathrm{NiO}$ phases. However, the similar lattice parameters of $\mathrm{NiO}(0.418 \mathrm{~nm}), \mathrm{FeO}(0.433 \mathrm{~nm})$ and $\mathrm{CoO}(0.509 \mathrm{~nm})$ - in $\mathrm{NaCl}$ configuration for example - are not considered in this context. EDS maps with high spatial resolution $<0.5 \mathrm{~nm}$ (Fig. 1c) reveal that Fe, Ni and Co are distributed similarly while the Ce precipitates in different locations. While the bulk average composition was confirmed to be $\mathrm{Ni}_{0.3} 0 \mathrm{Fe}_{0.07} \mathrm{Co}_{0.20} \mathrm{Ce}_{0.43} \mathrm{O}_{\mathrm{x}}$, EDS measurements on the $10 \mathrm{~nm}$ scale reveal significant compositional variation. For example, the observed composition for the region in Figure $1 \mathrm{c}$ is $\mathrm{Ni}_{0.28} \mathrm{Fe}^{0.20} \mathrm{Co}_{0.10} \mathrm{Ce}_{0.34} \mathrm{O}_{\mathrm{x}}$. Our investigations are compatible with a microstructure where the transition metal oxides form miscible agglomerates of nanocrystals to create a matrix that encloses isolated $\mathrm{CeO}_{2}$ precipitates. A preparation and calcination of the material on glassy carbon support does not alter this nanostructure but stimulates the growth of graphitic carbon (Figure 1d). Finally, the Figure 1e) shows that the nanostructure of the matrix can be tuned by growth processes: Electrodeposition of $\mathrm{Ni}_{0.30} \mathrm{Co}_{0.20} \mathrm{Ce}_{0.5} \mathrm{O}_{\mathrm{x}}$ reduces the grain sizes in the matrix so much that if becomes locally undistinguishable from an amorphous structure while the size and the crystallinity of the $\mathrm{CeO}_{2}$ precipitates are preserved. [4]

Rferences:

[1] J.A. Haber et al., Energy Environ. Sci. 7 (2014) 682-688

[2] B. Barton et al. Microsc.Microanal. 18 (2012) 1606-1607

[3] C. Kisielowski et al., Phys. Rev. B 88 (2013) 024305

[4] This work is performed by the Joint Center for Artificial Photosynthesis, a DOE Energy

Innovation Hub, supported through the Office of Science of the U.S. Department of Energy under 
Award Number DE-SC000499. Electron Microscopy was performed at the NCEM, which is supported by the Office of Science, Office of Basic Energy Sciences of the U.S. Department of Energy under Contract No. DE-AC02-05CH11231.

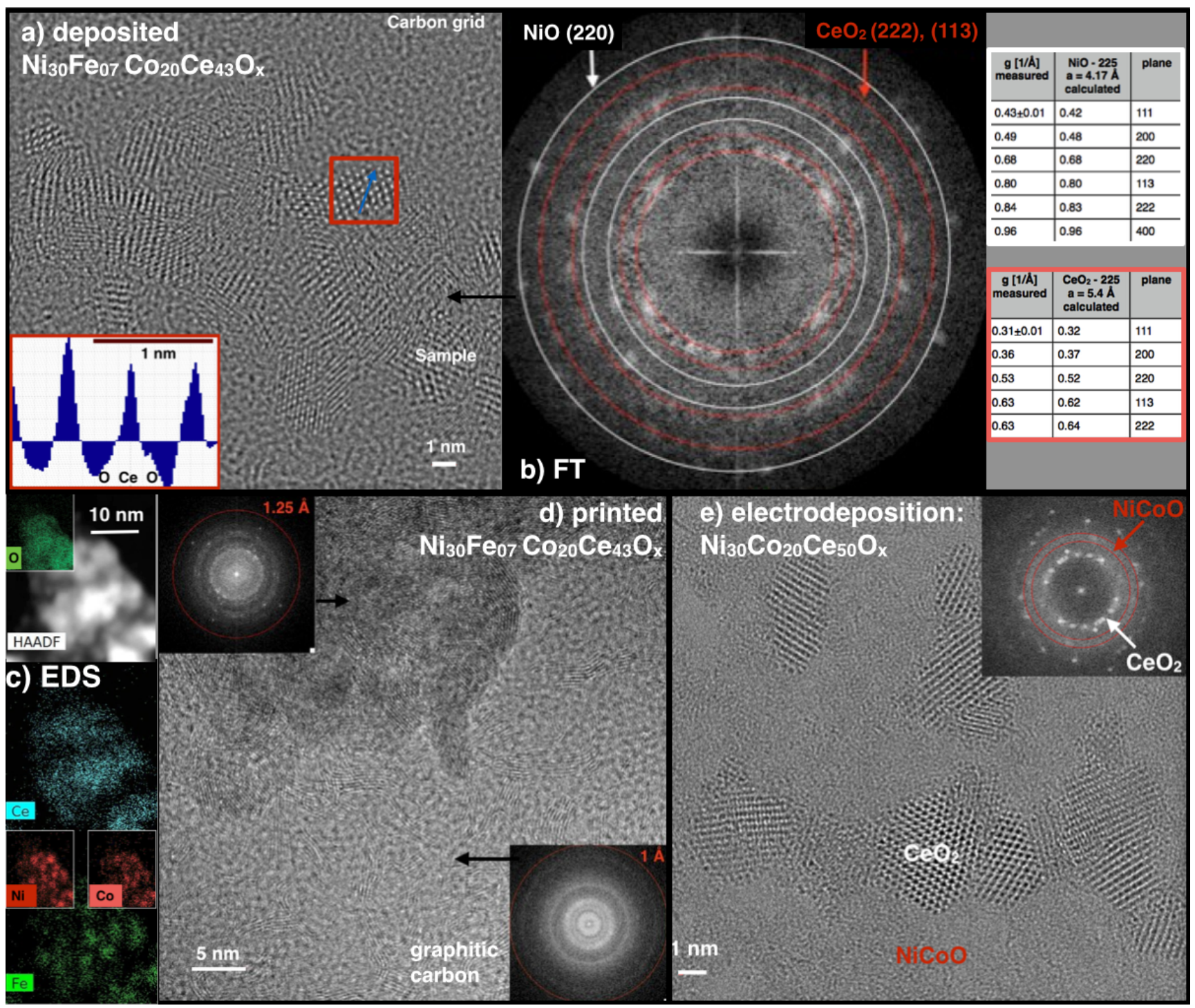

Figure 1. Structural analysis of new, quaternary $\mathrm{Ni}_{a} \mathrm{Fe}_{b} \mathrm{Co}_{\mathrm{c}} \mathrm{Ce}_{0 \mathrm{~d}} \mathrm{O}_{\mathrm{x}}$ catalysts for oxygen evolution. Details of the frames a) - e) are described in the text. 